\title{
Iris recognition algorithm based on Contourlet Transform and Entropy
}

\author{
Ezzaki Ayoub, ${ }^{\mathrm{a}}$ Idrissi Zouggari Nadia, ${ }^{\mathrm{a}}$ Francisco-Angel Moreno ${ }^{\mathrm{b}}$ Masmoudi Lhoussaine, ${ }^{\mathrm{a}}$ \\ ${ }^{a}$ LCS laboratory, Physics Dept. Faculty of science, Mohammed V University in Rabat, Morocco. \\ b Machine Perception and Intelligent Robotics Group, System Engineering and Automation Dept. and \\ Biomedical Research Institute of Malaga (IBIMA), University of Malaga, Malaga, Spain.
}

Received 30rd of August 2019; accepted $24^{\text {th }}$ of July 2020

\begin{abstract}
The iris is one of the most secure biometric types of information that is widely employed in authentication systems. In this paper we present a method for iris recognition based on the Contourlet Transform and Shannon Entropy which entails (i) the detection and segmentation of the iris, (ii) its normalization, (iii) the application of the Contourlet Transform, (iv) the generation of the iris descriptor, and (v) the matching between the query iris and those in the database. The proposed method has been tested with images taken from the popular CASIA-V4 and UBIRIS.v1 datasets and compared against other six recent iris recognition algorithms using the statistics EER, AUC and ARR. The results show a higher performance of the proposed method with a reduced computation time.
\end{abstract}

Keywords: Iris, Biometric, Contourlet Transform, entropy, Segmentation, Hough transform

\section{1- Introduction}

Biometric information is unique for every person, being different even for twins. For this reason, it is used in the majority of security and surveillance systems, as well as being widely employed nowadays in a lot of our daily use devices (e.g. phones, cars, TVs, doors, etc.), taking advantage of its physiological, non-invasive and passive characteristics.

In particular, iris recognition has become, in the last two decades, one of the most used biometrics in authentication systems, significantly increasing the efficiency and performance of recognition systems. Biologically speaking, the iris represents the colored muscle bounded by the pupil (Figure 1), which changes its size by the contraction and dilatation of the iris. This part of the eye contains a random and unique pattern of microstructures that can be used as a characteristic signature for person identification. This signature, though, can be disturbed by the upper and lower eyelids and even by the cornea [1]. Iris detection and recognition was first introduced by Flom [2] in 1987 and subsequently further explored in several research works that applied this approach to a wide range of applications [3][6]. For instance, in the work [7] Rai \& al. proposed an algorithm for iris detection based on Support Vector Machines (SVM) and the Hamming distance. For the same purpose, Patil \& al. [8] applied a $6^{\text {th }}$ level of wavelet approximation. Other work proposed by Li Ma \& al. [9] proposed the use of the Hough transformation and spatial filters for features extraction. Another recent work based on Displacement Information that uses a Sparse Matching Technique was proposed by Iglesias \& al. in [10]. Finally,

Correspondence to: ayoub_ezzaki@um5.ac.ma

Recommended for acceptance by Angel D. Sappa

https://doi.org/10.5565/rev/elcvia. 1190

ELCVIA ISSN: 1577-5097

Published by Computer Vision Center / Universitat Autonoma de Barcelona, Barcelona, Spain 
different comparative studies have been published [11]-[13], identifying the presence of the eyelids and image noise, as well as an excessive processing time, as the main issues affecting the performance of the iris detection and recognition.

In this paper, we propose an algorithm for iris recognition based on the Contourlet Transform (CT) and the Shannon Entropy, which can be divided into five steps:

1- Iris detection and segmentation.

2- Iris normalization.

3- Contourlet Transform application.

4- Features extraction.

5- Template matching.

As a contribution, the proposed method avoids the use of the complete iris information by wisely choosing the parts with higher entropy before generating a unique code through the application of the $\mathrm{CT}$, in order to minimize the recognition cost, which might be excessive especially in large databases. In addition, our proposal presents a higher performance score (based on Equal Error Rate (EER), Area Under the receiver operating characteristics Curve (AUC) and Accuracy Recognition Rate (ARR) metrics) than other works presented in the literature, while keeping the processing time short enough to be usable in most real time applications, hence revealing its potential as an alternative method for iris recognition systems. Finally, and in order to assess the performance of the proposed method, a series of experimentations has been carried out using three sets of iris images taken from the CASIA-IrisV4 [14] and UBIRIS VI [15] datasets, which include images with and without noise, and also images captured from twins.

The outline of this paper is as follows: we first describe in Section 2 the iris databases employed in this work. In section 3, we provide all the details of the proposed algorithm step by step, while section 4 presents a set of experiments designed to evaluate the performance of our proposal in different cases in comparison with other state-of-the-art iris recognition systems. Finally, we provide some conclusions about the proposed method in section 5 .

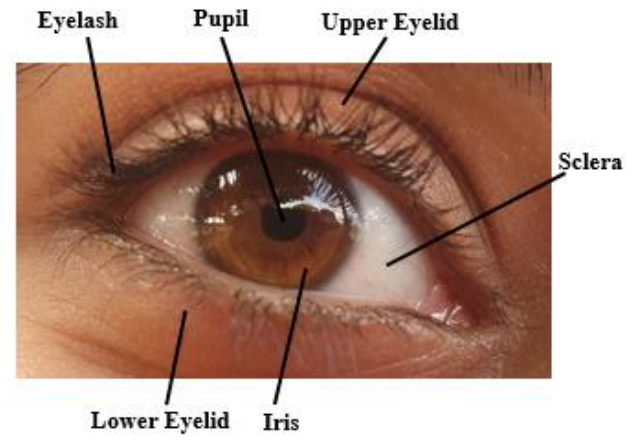

Figure 1: Front view of human eye

\section{2- The iris databases}

In order to assess the performance of the proposed algorithm, we have employed the CASIA dataset [14] provided by the Institute of Automation of the Chinese Academy of Science, and the UBIRIS.v1 dataset [15] from the SOCIA Lab - Soft Computing and Image Analysis Group.

CASIA is one of the most widely employed iris databases, whose latest version, named CASIAIrisV4, was published in 2010 and is composed by six subsets: CASIA-Iris-Interval, CASIA-Iris-Lamp, CASIA-Iris-Twins, CASIA-Iris-Distance, CASIA-Iris-Thousand and CASIA-Iris-Syn. It provides more than $54 \mathrm{k}$ grayscale images taken under near infrared illumination (Figure 2-a) to allow the study of the iris texture, also includes a subset with images with iris from twins (Figure 2-c). The second database, 
UBIRIS.v1, is composed of 1877 noisy iris images collected from 241 people (Figure 2-b), which can be used to test the algorithm robustness to the presence of noise.

The choice of these two databases was directed by the need of non-ideal iris images to evaluate the robustness of our proposal, as the images in the CASIA database present both eyelids and eyelashes that cover part of the iris, hence becoming a more challenging dataset, while the images in the UBIRIS database are corrupted with noise.

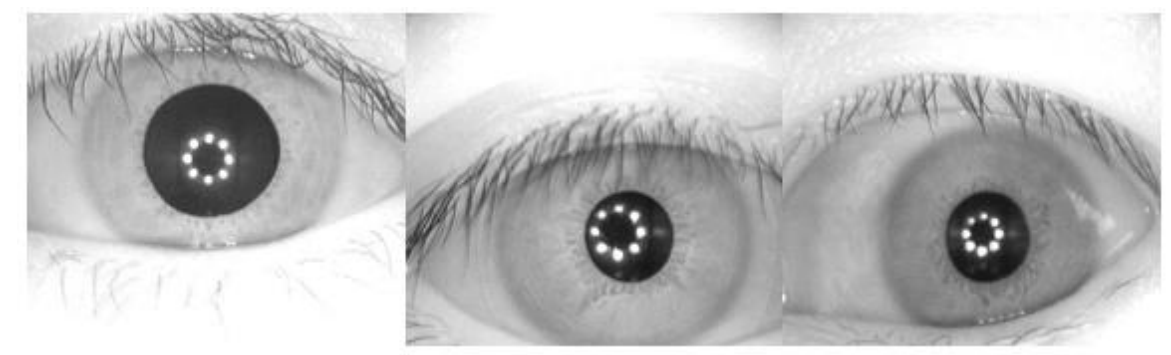

(a)

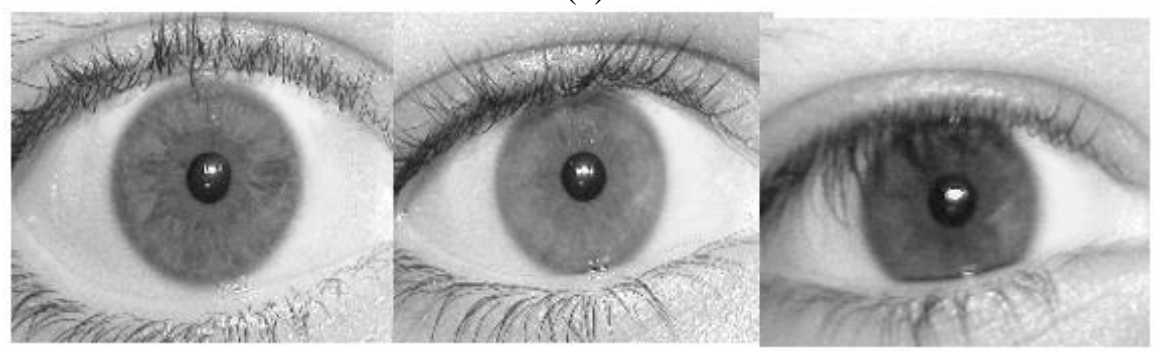

(b)

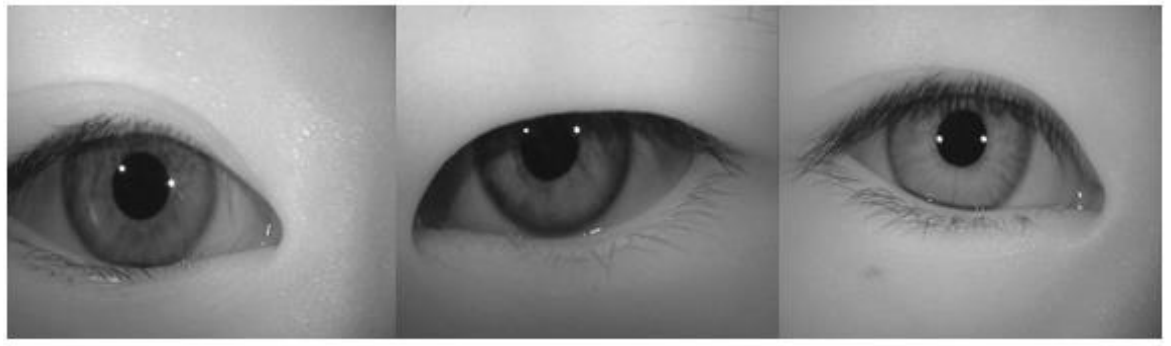

(c)

Figure 2: Iris images from (a) CASIA-Iris-Interval database. (b) UBIRIS.v1 database. (c) CASIA-IrisTwins database.

\section{3- Proposed Algorithm}

\section{1- Iris detection and segmentation}

This is the first step of the procedure and involves: i) detecting and removing the pupil, ii) detecting and suppressing both the eyelashes and the eyelids, and iii) extracting the iris region, as explained next.

The pupil represents the darkest part of the human eye, whose size ranges from $1.5 \mathrm{~mm}$ to $8 \mathrm{~mm}$ [16] and which generally is located in the center of the eye (except in some pathological cases like Corectopia). Besides, it contains, in most of the databases, the light reflection from the acquisition system. The first step in the process of iris detection involves the detection and masking of the pupil in the image, which can be accomplished through a binarization process that exploits its high intensity nature. This process is widely used in different kind of image processing algorithms [17], [18], but it can be easily affected by a unbalanced image illumination and even with the degradation of the image quality. 
A classical global thresholding method was proposed by Otsu in [19] using the intensity distribution of an image. In [20], Chen \& al [20]. proposed a multiple stage algorithm based on edge detection and the intensity information of the pixels. The authors in [21] proposed a global thresholding algorithm using integral images and the use of the mode. Another adaptive thresholding algorithm was proposed in [22], which removes the background using the mean deviation and the local mean. Finally, Singh \& al [23] proposed a method which finds suitable binarization thresholds for a given image based on the local properties and the position of the pixel. These algorithms can be divided into two categories:

- Global thresholding algorithms: Even with an easy implementation and low computational time, this type of binarization algorithms are sensible to noise and struggle with low quality images.

- Local thresholding algorithms: This group can handle uneven illumination variations, but with a significant processing cost.

Local thresholding algorithms are more suitable for applications like iris recognition, where the quality of the captured image is not always satisfying. For this purpose, we applied three local thresholding methods (Chen \& al, Singh \& al and Romen \& al. ) on 300 iris images taken from the dataset presented in [24]. The performance of the three methods is evaluated on the totality of the dataset and compared to its ground-truth using the three metrics:

- Peak Signal to Noise Ratio (PSNR): denotes how much the processed image is similar to the ground-truth [25].

- Misclassification Penalty Metric (MPM): evaluates the binarization process against the ground truth. Misclassified pixels are penalized based on their distances from the ground truth reference object's border [26].

- Misclassification Error (ME): present the percentage of object pixels wrongly assigned as background pixels [27].

Figure 3 shows an example of the application of the three evaluated local thresholding methods on two iris sample images taking from the mentioned dataset (Image number 2249 and 3561), compared to the ground-truth image.
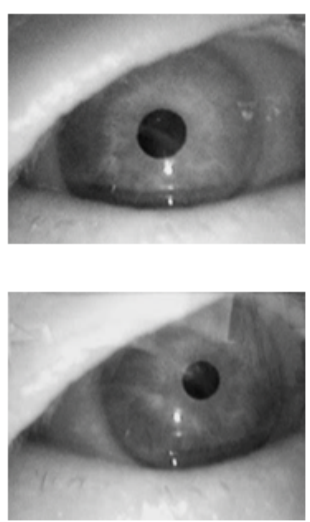

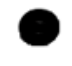

(a)

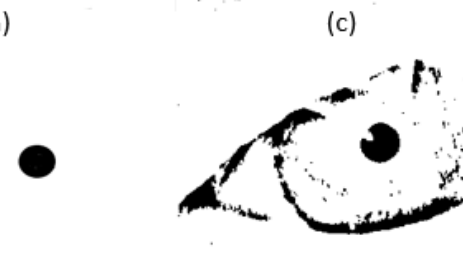

(b)

(d)

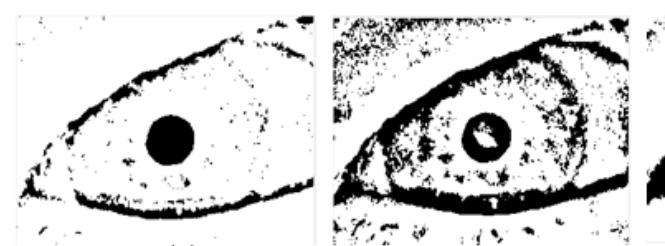

(e)

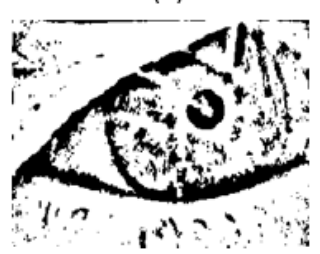

(f)

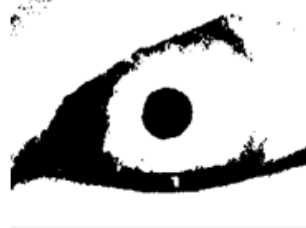

(g)

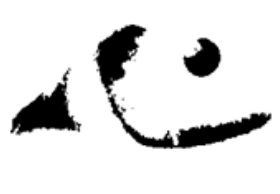

(h)

Figure 3: Binarization process compared to the ground-truth, using three different methods: a, b) Ground-truth, c, d) Singh \& al [23]. e, f) Romen \& al. [22]. and g, h) Chen \& al. [20]. 
Table 1: PSNR, MPM, ME metrics and processing time for the tested binarization methods.

\begin{tabular}{ccccc} 
& PSNR & MPM & ME & $\begin{array}{c}\text { Processing time } \\
\text { (Resolution 640 } \times \mathbf{4 8 0} \text { ) }\end{array}$ \\
\hline Chen \& al. [20] & 17.13 & 1.78 & 15 & $1.21 \mathrm{sec}$ \\
Singh \& al. [23] & $\mathbf{1 9 . 9 1}$ & $\mathbf{3 . 0 8}$ & $\mathbf{1 0}$ & $\mathbf{0 . 4 6 ~ s e c}$ \\
Romen \& al. [22] & 18.80 & 1.23 & 14.2 & $0.66 \mathrm{sec}$ \\
\hline
\end{tabular}

As can be seen in Table 1, which shows the PSNR, MPM, ME and the processing time of the three methods, the results given by Singh \& al's method are the most satisfying in term of binarization quality for pupil removal. In addition, it presents an optimal processing time compared to the other tested methods, which is an important factor when developing in such real time applications. Given these results, we have adopted this method for our application.

Once the pupil has been removed, we address the suppression of the eyelashes and the eyelids, which, in general, occlude the top and down parts of the iris region. We have employed the method proposed by Naguru [28], which eliminates the undesired parts on the upper and lower boundaries of the iris using the edge map of the gradient, and the hyperbolic Hough Transform. HT is a technique that can be used to isolate features of a particular shape [29]. The main motivation of using this technique is that the eyelashes are typically almost vertically aligned on an hyperbolic shape, so we can take only the vertical gradient points (instead of the original gray scale image) with the hyperbolic HT which has less parameters than other techniques presented for the same purpose, hence reducing the effect of the eyelids and the eyelashes when applying the circular Hough transform for iris segmentation, while reducing at the same time the processing cost of the system [11], as shown in Figure 4.

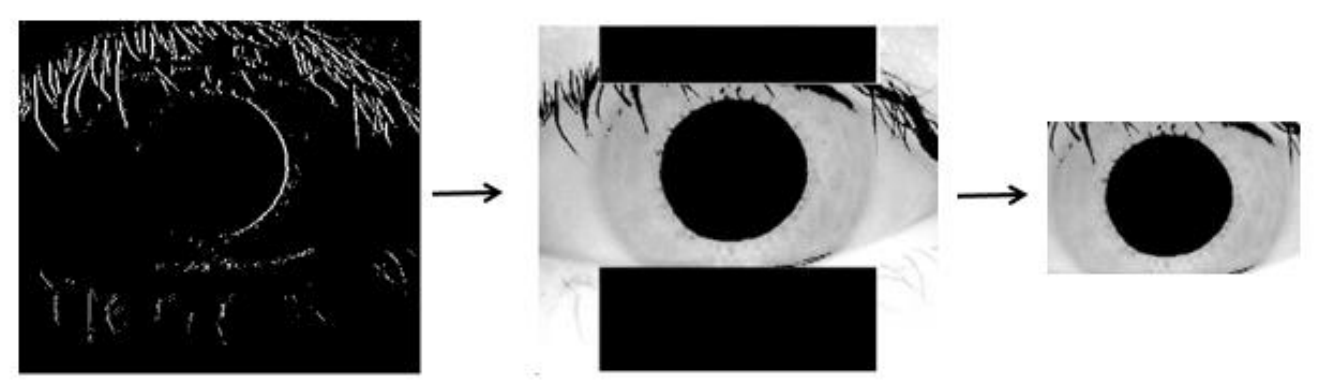

Figure 4: Eyelids and eyelashes elimination at the top and the bottom of the iris region.

Next, once the non-relevant parts of the image have been deleted, we deal with the iris segmentation process through a combination of (i) an edge detection algorithm based on the quantum superposition law, that we proposed in [30], which achieves better performance than other edge detectors in terms of edge continuity even with the presence of noise, and (ii) the circular Hough Transform [31]. This combined process yields the center position $(a, b)$ and the radius $r$ of both the pupil and the iris.

It is important to note that the range of radii to be found in the image depends on the employed database, being, for the CASIA database, a range between 90 and 150 pixels for the iris, and between 28 to 75 pixels for the pupil [11]. Figure 5 shows the extracted iris region in an example image from the CASIA dataset. 


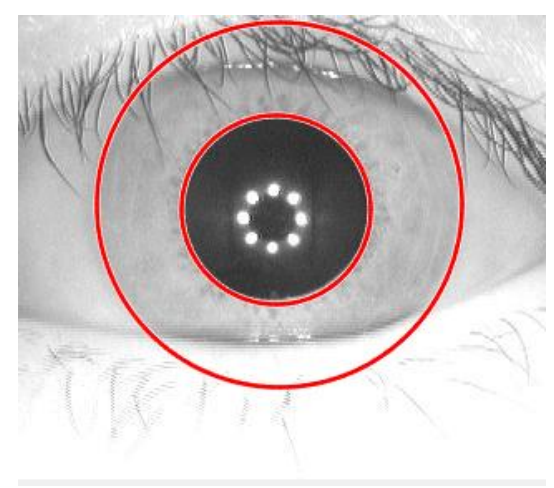

Figure 5: Iris detection.

Even with its satisfying performance in the majority of cases, the HT method fail to deal with some special cases. The Figure 6 presents an example of the most typical failure types which are:

- A dark image (unbalanced illumination) (Figure 6.a).

- A squeezed eye (Figure 6.b).

- The eyelids match circular module (Figure 6.c).

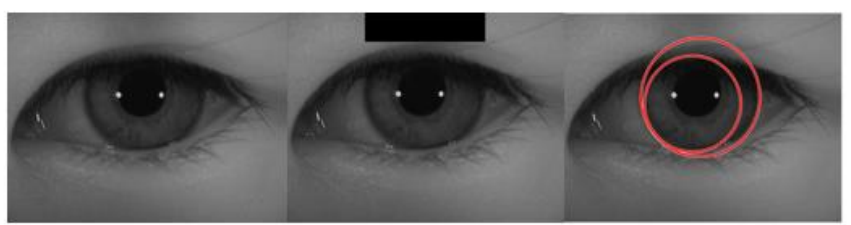

(a)

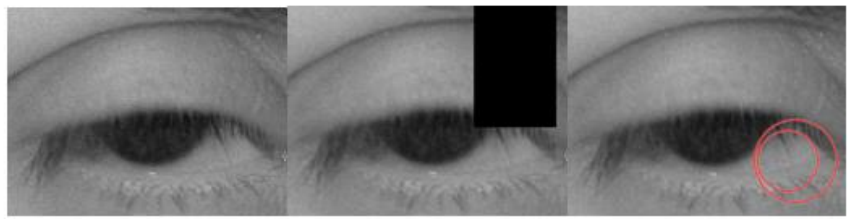

(b)

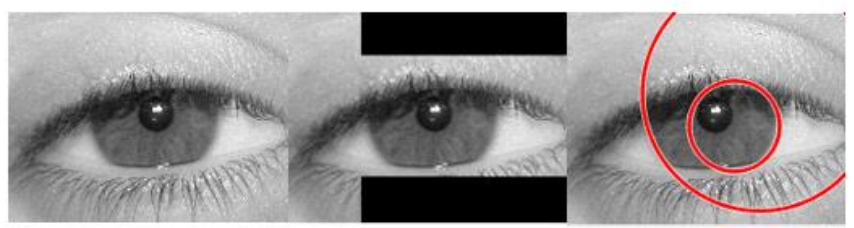

(c)

Figure 6: Typical failure types: a) Dark image (unbalanced illumination. b) Eyelid match circular module. c) Squeezed eye.

These anomalies affect the quality of the iris detection process, which subsequently affects the recognition step of the algorithm.

\section{2- Iris normalization}

Now, in order to extract the most significant part of the iris regardless its size, we have applied the Daugman's rubber sheet model [32], which maps each point from the iris region to a rectangular area (Figure 7) with polar coordinates $(r \in[0,1], \theta \in[0,2 \pi])$. Thus, the transformation of the iris region image $I(x, y)$ is given by a map:

$$
I(x(r, \theta), y(r, \theta)) \rightarrow I(r, \theta)
$$


with:

$$
\begin{aligned}
& x(r, \theta)=(1-r) x_{p}(\theta)+r x_{i}(\theta) \\
& y(r, \theta)=(1-r) y_{p}(\theta)+r y_{i}(\theta)
\end{aligned}
$$

and where $\left(x_{p}, y_{p}\right)$ and $\left(x_{i}, y_{i}\right)$ represent the iris and the pupil boundaries coordinates in the direction $\theta$, respectively. This model also takes into consideration the pupil dilation in order to give a constant normalized iris.

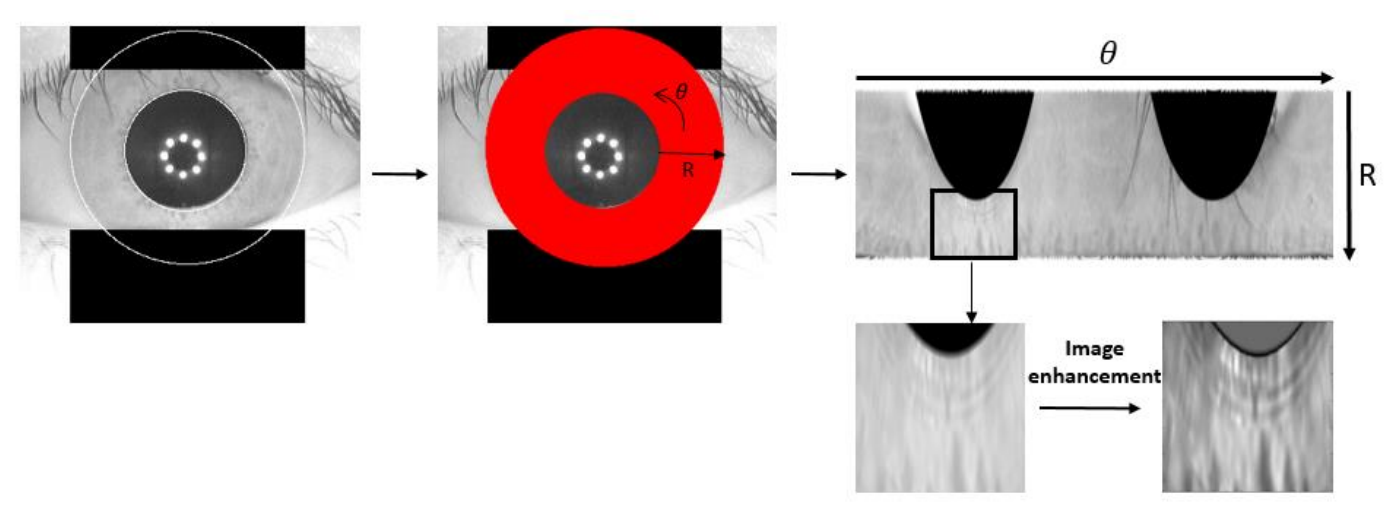

Figure 7: Iris normalization and enhancement.

Finally, in order to take into account only the information from the iris, the black areas of the normalized image are discarded, while the iris region goes through an enhancement process using the method based on the quantum superposition law that we presented in [30]. This corrects the illumination irregularities and enhances the intensity discontinuities, which will be useful for the generation of the iris descriptor from.

\section{3- Contourlet Transform application.}

Since the iris is different for every person, we want to extract a signature (or descriptor) that makes unique the information extracted from it. To achieve this, we have used the so-called Contourlet Transform (CT), which was proposed by Do \& Vetterli in 2002 [33], and which performs a multiresolution, local and directional image expansion through a set of filter banks as shown in Figure 8. Thanks to its directionality and anisotropy properties, the CT can present the iris information with a small number of coefficients. 


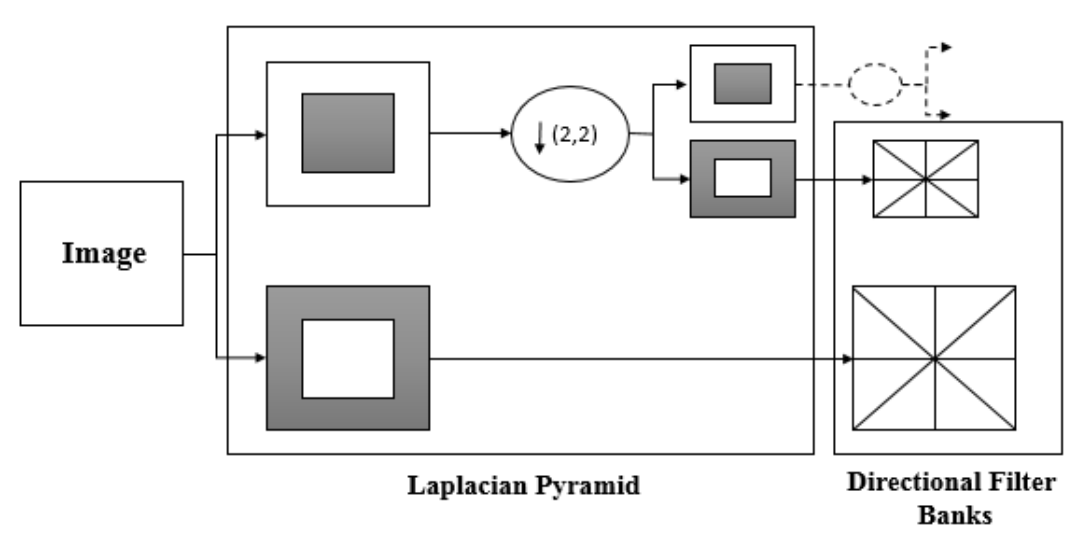

Figure 8: Contourlet Transform.

The process is as follows: the original iris image is represented as a set of multiple band-passed images, using a Laplacian pyramid [34] that generates at each level of decomposition a down sampled low pass image $(a)$ and a band-pass image $(b)$, which is the difference between the original image and the prediction (Figure 9), so that point discontinuities, presented by the iris tiles, can be detected. After that, we apply a set of directional filter banks DFB [35] in order to capture high frequency content as, for example, contours and edge discontinuities [36], which represent, in our case, the iris texture patterns. These steps are repeated for each band-passed in the pyramid image to obtain the multidirectional and multi-resolution decomposition of the image (Figure 10).

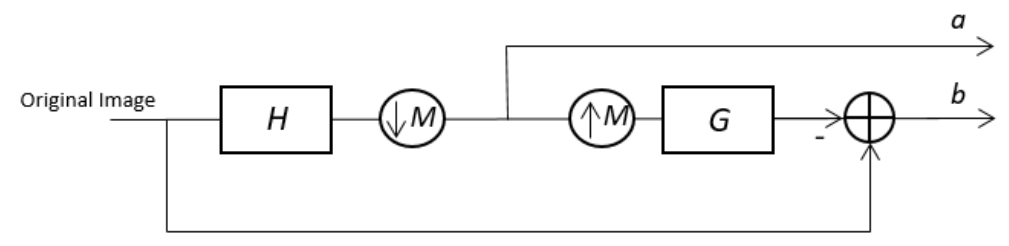

Figure 9: Laplacian pyramid.

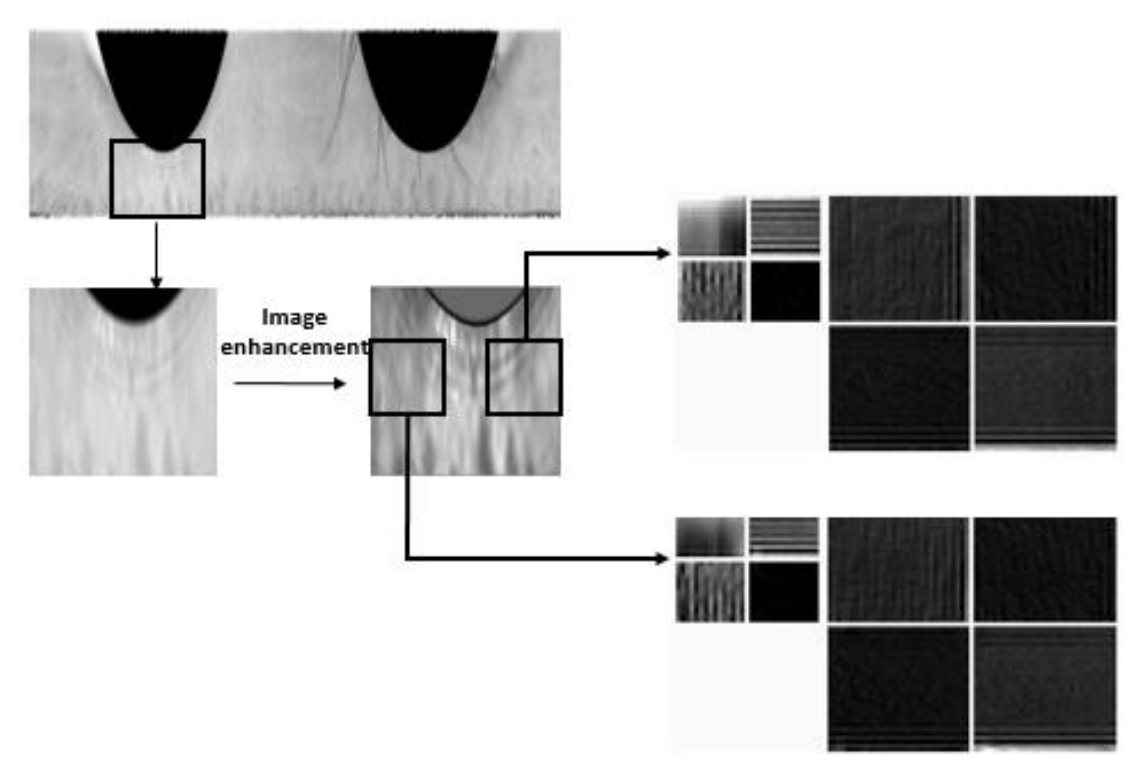

Figure 10: Contourlet Transform for an iris block. 


\section{4- Features extraction}

Since applying the CT to the whole iris image would provide too much information (some of it either redundant or not significant), we aim to extract only the most significant piece of information so that we can increase the correct recognition rate while, at the same time, reducing the computation time. For that, we have selected a set of blocks in the image that exhibit a high entropy [37] value in order to maximize the amount of information they provide and to avoid the parts with no significant iris information (black pixels). Thus, we apply the CT to four blocks of $32 \times 32,64 \times 64$ or $128 \times$ 128 pixels (the best block size will be chosen in the experiment results section), one in each orthogonal direction (Figure 11), in order to find a compact descriptor that encompasses as much information as possible in both the adequate frequency band and in different iris directions. Finally, the frequency bands are converted to an iris descriptor (see Figure 12) that will be used in the recognition process.

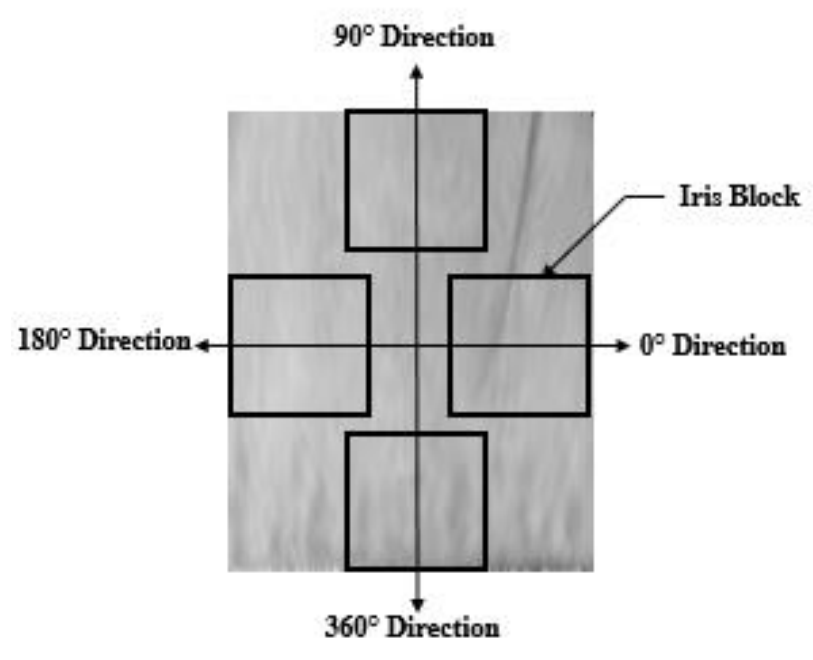

Figure 11: Extraction of four iris blocks in the 4 directions.
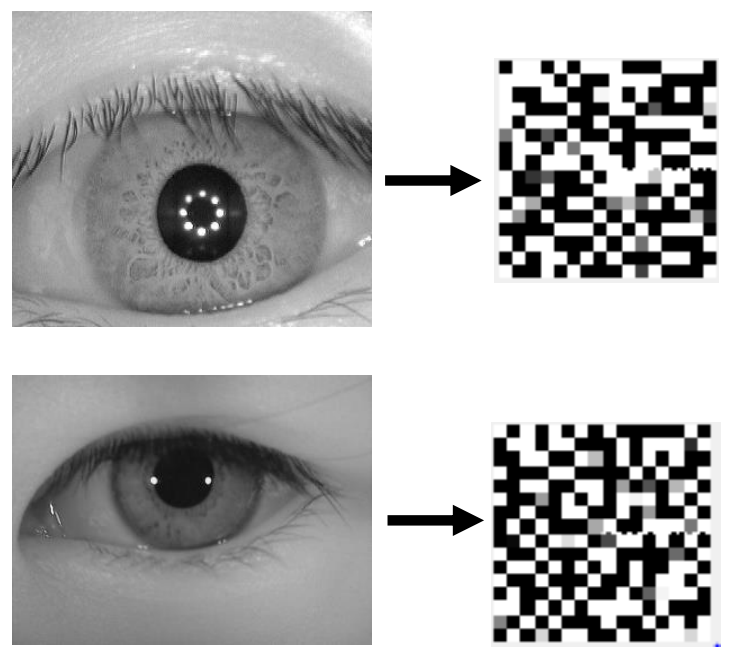

Figure 12: Extracted features for two different iris images. 


\section{5- Template matching.}

With all the above explained process, we have created a unique descriptor that represents the iris unambiguously. This descriptor can be used to make a decision by finding a corresponding iris within a database of iris descriptors. For that, we compute the Euclidean distance, which achieves good results in biometric recognition systems, especially regarding iris and facial characteristics [38], [39]. Using the Euclidean distance is a general matching approach, typically employed in mathematical geometry, which yields a distance of zero for an identical match between the query iris and those in the database.

As a summary, all the steps of the proposed algorithm is presented on the diagram below:

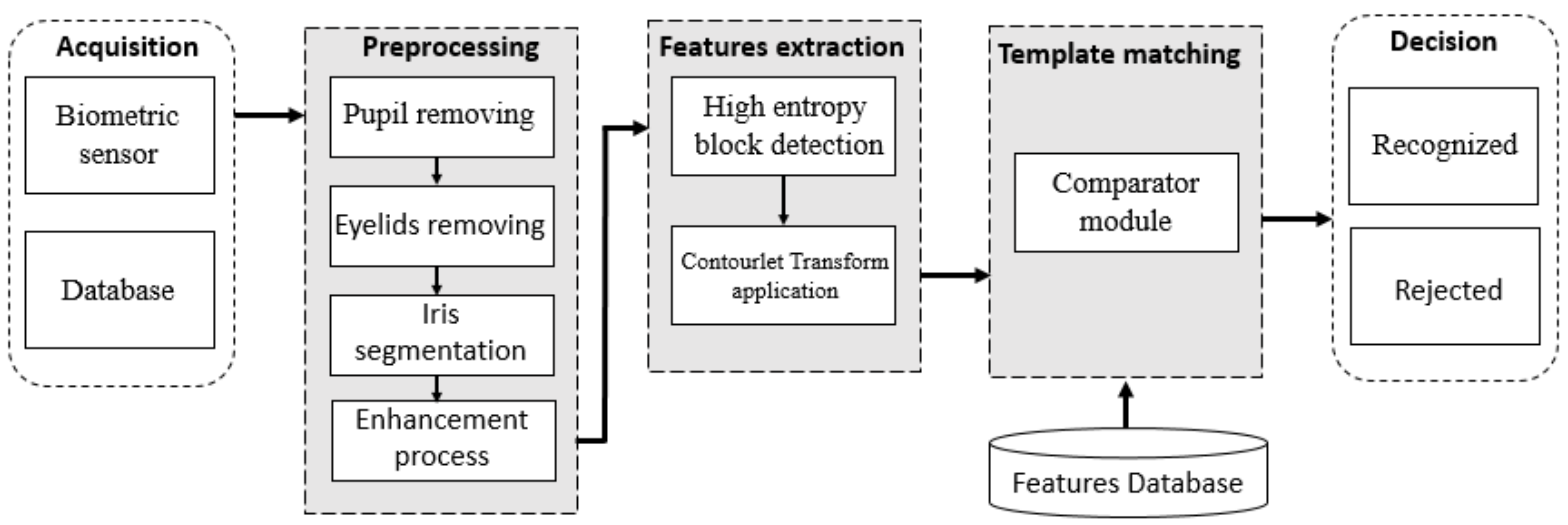

Figure 13: Diagram of the proposed method.

\section{4- Experimental results.}

In order to test the proposed algorithm, we first apply it with the CASIA-Iris-Interval database, which contains the iris images of 249 subjects with a total of 2639 images [14], and then with the UBIRIS database, containing 1877 noisy images collected from 241 eyes [15]. We have compared the results with the method proposed by Masek [11], Daugmana [32], Radu [40], Mira [41] and Radman [42] using the EER, AUC and ARR statistics. The results are shown in Tables 3 and 4. Before that, an experimentation using different block sizes (as mentioned in Section 3.4) is carried out to empirically find the adequate block size for this application. The idea is to find a compromise between having sufficient information to generate a unique iris code while incurring in minimum computational time and process cost. Table 2 below shows the recognition rate of our proposal, for the CASIA iris Interval database, using different block sizes.

Table 2: Recognition rate using different block sizes.

\begin{tabular}{cccc}
\hline CASIA-Iris- Interval & $32 \times 32$ & $64 \times 64$ & $128 \times 128$ \\
\hline Recognition Rate & 97.12 & $\mathbf{9 9 . 8 7}$ & 95.89 \\
\hline
\end{tabular}

Given the results, we have used $64 \times 64$ blocks for the rest of the experiments, which gives the best results in term of recognition rate.

Table 3: Comparison between the proposed method and other algorithms applied on CASIA Database.

\begin{tabular}{ccccc}
\hline CASIA-Iris- Interval & Masek [11] & Daugmana [32] & Mira [41] & Proposed method \\
\hline EER \% & 5.60 & 1.05 & 1.04 & $\mathbf{0 . 0 9}$ \\
ARR \% & 90.09 & 98.95 & 98.88 & $\mathbf{9 9 . 8 7}$ \\
AUC & - & $\mathbf{0 . 9 9 7}$ & 0.998 & 0.999 \\
\hline
\end{tabular}


Table 4: Comparison between the proposed method and other algorithms applied on UBIRIS

Database.

\begin{tabular}{cccc}
\hline UBIRIS & Radu [40] & Radman [42] & Proposed method \\
\hline EER \% & 1.80 & 9.48 & $\mathbf{1 . 2 1}$ \\
ARR \% & 99.23 & 97.36 & $\mathbf{9 9 . 5 2}$ \\
\hline
\end{tabular}

Besides, since the iris is an epigenetic phenotypic feature (which means that even twins have their unique iris information), the matching process should also work with image of iris captured from twins. This case has also been tested with the CASIA-Iris-Twins database (Figure 14), which contains iris images of 100 pairs of twins [14]. The table below show the ARR value of the proposed algorithm compared to the algorithm proposed by Mohd [43] applied on the CASIA twin database.

Table 5: Comparison between the proposed method and Mohd [43] algorithms applied on CASIA Twins Database.

\begin{tabular}{ccc}
\hline CASIA-Iris-Twins & Mohd [43] & Proposed method \\
\hline EER \% & - & $\mathbf{1 . 2 1}$ \\
ARR \% & 99.47 & $\mathbf{9 9 . 8 1}$ \\
\hline
\end{tabular}

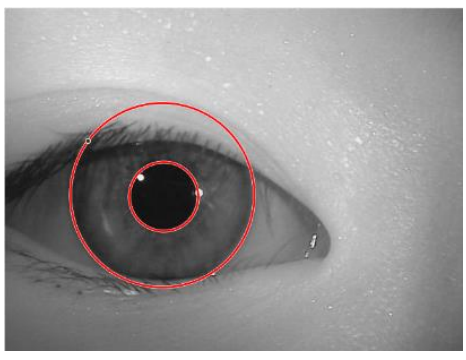

(a)

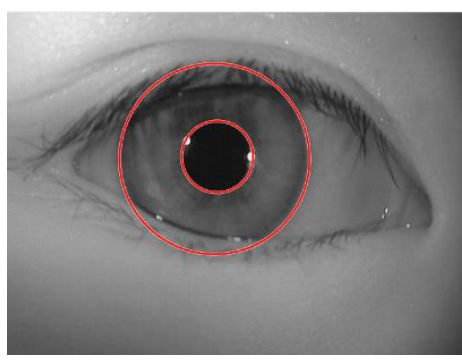

(b)

Figure 14: Segmented iris images of two twins (CASIA-Iris-Twins Database).

Finally, a system based on iris detection and recognition, apart from being robust, should also run in a time short enough to be usable for real applications, i.e. the computational time should be as low as possible. In our tests, our proposal recognizes an iris from a database of 60 images in 3.16 seconds, including all stages of the process, using MATLAB $2018 \mathrm{~b}$ in an Intel Xeon CPU, $3.50 \mathrm{GHz}$ with $8 \mathrm{~GB}$ of RAM, divided as follow:

Table 6: Computational time of each part of the proposed method.

\begin{tabular}{lc}
\hline \multicolumn{1}{c}{ Task } & $\begin{array}{c}\text { Average Time } \\
\text { (in Seconds) }\end{array}$ \\
\hline Pupil removing & 0.31 \\
Eyelids removing & 0.38 \\
Iris segmentation & 0.92 \\
Enhancement process & 0.50 \\
Features extraction & 0.25 \\
Template matching & 0.80 \\
\hline & \\
\cline { 2 - 2 } & Total: $\mathbf{3 . 1 6} \mathbf{~ S}$ \\
\hline
\end{tabular}




\section{5- Conclusion}

In this paper we have presented an algorithm for iris recognition that is robust against noise and which outperforms the performance of other recent techniques in the literature. Its computation burden is low enough to make it usable in real applications. The proposed algorithm analyzes the detected iris in order to extract a unique iris signature using the Contourlet Transform and the Shannon Entropy. The generated descriptor can be then compared against a database of iris codes through the Euclidean distance, yielding a matching score employed to make a decision about the existence or not of the iris in the database.

To situate our work to other researches, we have made a comparative study with six other algorithms for iris recognition, using EER, ARR and AUC statistics, and a set of popular, publicly available, iris datasets achieving highest performance at a reduced computational time even with images affected by noise. Our proposal has also been validated as part of a secure access system for our agricultural robots we present in [44]. As future work, we will exploit the obtained results to design an electronic card specifically dedicated to a multiuser authentication system, for agricultural applications, based on iris recognition.

\section{ACKNOWLEDGMENT}

Authors are thankful to the Ministry of Higher Education and Scientific Research of Morocco (MESRSFC), and the National Center for Scientific and Technical Research of Morocco (CNRST) for financing this project, and to the Institute of Automation, Chinese Academy of Science for their efforts in making CASIA iris database, and for SOCIA Lab. - Soft Computing and Image Analysis Group for making the UBIRIS database for their efforts on the construction of a noised iris database for iris detection and recognition applications.

\section{Reference}

[1] A. Matin, F. Mahmud, S. T. Zuhori, and B. Sen, "Human iris as a biometric for identity verification," in 2016 2nd International Conference on Electrical, Computer Telecommunication Engineering (ICECTE), Dec. 2016, pp. 1-4, doi: 10.1109/ICECTE.2016.7879610.

[2] L. Flom and A. Safir, "Iris recognition system," Feb. 20, 1985.

[3] C.-L. Tisse, L. Martin, L. Torres, and M. Robert, "Person Identification Technique Using Human Iris Recognition," Vis. Interface VI2002, pp. 294-299, 2002.

[4] A. F. Abate, M. Frucci, C. Galdi, and D. Riccio, "BIRD: Watershed Based IRis Detection for mobile devices," Pattern Recognit. Lett., vol. 57, pp. 43-51, May 2015, doi: 10.1016/j.patrec.2014.10.017.

[5] D. Gragnaniello, C. Sansone, and L. Verdoliva, "Iris liveness detection for mobile devices based on local descriptors," Pattern Recognit. Lett., vol. 57, pp. 81-87, May 2015, doi: 10.1016/j.patrec.2014.10.018.

[6] Y. Chen, Y. Liu, and X. Zhu, "Robust iris segmentation algorithm based on self-adaptive ChanVese level set model," J. Electron. Imaging, vol. 24, no. 4, 2015, doi: 10.1117/1.JEI.24.4.043012.

[7] H. Rai and A. Yadav, "Iris recognition using combined support vector machine and Hamming distance approach," Expert Syst. Appl., vol. 41, no. 2, pp. 588-593, Feb. 2014, doi: 10.1016/j.eswa.2013.07.083.

[8] C. M. Patil and S. P. Kulkarni, "An Approach of Iris Feature Extraction for Personal Identification," in 2009 International Conference on Advances in Recent Technologies in 
Communication and Computing, Kottayam, Kerala, India, 2009, pp. 796-799, doi: 10.1109/ARTCom.2009.14.

[9] Li Ma, Tieniu Tan, Yunhong Wang, and Dexin Zhang, "Efficient iris recognition by characterizing key local variations," IEEE Trans. Image Process., vol. 13, no. 6, pp. 739-750, Jun. 2004, doi: 10.1109/TIP.2004.827237.

[10] P. Iglesias, R. Hernández-García, R. J. Barrientos, E. Goncalves, and M. Mora, "Iris Recognition based on Displacement Information using a Sparse Matching Technique," in 2019 38th International Conference of the Chilean Computer Science Society (SCCC), Nov. 2019, pp. 1-8, doi: 10.1109/SCCC49216.2019.8966438.

[11] L. Masek, "Recognition of human iris patterns for biometric identification," 2003.

[12] S. V. Sheela and P. A. Vijaya, Iris Recognition Methods- Survey. .

[13] R. Vyas, T. Kanumuri, G. Sheoran, and P. Dubey, "Recent trends of ROI segmentation in iris biometrics: a survey," Int. J. Biom., vol. 11, no. 3, pp. 274-307, Jan. 2019, doi: 10.1504/IJBM.2019.100842.

[14] "Biometrics Ideal Test." http://biometrics.idealtest.org/dbDetailForUser.do?id=4 (accessed Feb. 21, 2018).

[15] H. Proença and L. A. Alexandre, "UBIRIS: A Noisy Iris Image Database," in Image Analysis and Processing - ICIAP 2005, Sep. 2005, pp. 970-977, doi: 10.1007/11553595_119.

[16] P. Reeves, "The Response of the Average Pupil to Various Intensities of Light," JOSA, vol. 4, no. 2, pp. 35-43, Mar. 1920, doi: 10.1364/JOSA.4.000035.

[17] Y. Min and Y. Zhang, "FADIT: Fast Document Image Thresholding," Algorithms, vol. 13, no. 2, Art. no. 2, Feb. 2020, doi: 10.3390/a13020046.

[18] L. Zhao, S. Zheng, W. Yang, H. Wei, and X. Huang, "An image thresholding approach based on Gaussian mixture model," Pattern Anal. Appl., vol. 22, no. 1, pp. 75-88, Feb. 2019, doi: 10.1007/s10044-018-00769-w.

[19] N. Otsu, "A Threshold Selection Method from Gray-Level Histograms," IEEE Trans. Syst. Man Cybern., vol. 9, no. 1, pp. 62--66, 1979.

[20] Q. Chen, Q. Sun, P. Ann Heng, and D. Xia, "A double-threshold image binarization method based on edge detector," Pattern Recognit., vol. 41, no. 4, pp. 1254-1267, Apr. 2008, doi: 10.1016/j.patcog.2007.09.007.

[21] W. Zhang and J. Dou, A Fast Thresholding Technique in Image Binarization for Embedded System. Vol 12(1) Pages 592-598, 2014. DOI: http://dx.doi.org/10.11591/telkomnika.v12i1.3359

[22] T. R. Singh, S. Roy, O. I. Singh, T. Sinam, and K. M. Singh, "A New Local Adaptive Thresholding Technique in Binarization,” vol. 8, no. 6, p. 7, 2011.

[23] B. Singh and A. P. Singh, "Edge Detection in Gray Level Images based on the Shannon Entropy 1," Journal of Computer Science, pp. 186-191, 2008.

[24] W. Fuhl, T. Kübler, K. Sippel, W. Rosenstiel, and E. Kasneci, "ExCuSe: Robust Pupil Detection in Real-World Scenarios," in Computer Analysis of Images and Patterns, vol. 9256, G. Azzopardi and N. Petkov, Eds. Cham: Springer International Publishing, 2015, pp. 39-51. 
[25] B. Bataineh, S. N. H. S. Abdullah, and K. Omar, "An adaptive local binarization method for document images based on a novel thresholding method and dynamic windows," Pattern Recognit. Lett., vol. 32, no. 14, pp. 1805-1813, Oct. 2011, doi: 10.1016/j.patrec.2011.08.001.

[26] D. P. Young and J. M. Ferryman, "PETS Metrics: On-Line Performance Evaluation Service," in 2005 IEEE International Workshop on Visual Surveillance and Performance Evaluation of Tracking and Surveillance, Beijing, China, 2005, pp. 317-324, doi: 10.1109/VSPETS.2005.1570931.

[27] M. Sezgin and B. Sankur, "Survey over image thresholding techniques and quantitative performance evaluation," J. Electron. Imaging, vol. 13, no. 1, pp. 146-165, Jan. 2004, doi: DOI: $10.1117 / 1.1631316$.

[28] I. Naguru and N. K. R. B, "Feature Matching in Iris Recognition System using MATLAB," Int. J. Adv. Sci. Eng. Inf. Technol., vol. 7, no. 5, pp. 1748-1757, Oct. 2017, doi: 10.18517/ijaseit.7.5.2765.

[29] T.-H. Min and R.-H. Park, "Eyelid and eyelash detection method in the normalized iris image using the parabolic Hough model and Otsu's thresholding method," Pattern Recognit. Lett., vol. 30, no. 12, pp. 1138-1143, Sep. 2009, doi: 10.1016/j.patrec.2009.03.017.

[30] A. Ezzaki, M. Lhoussaine, M. E. Ansari, F.-A. Moreno, R. Zenouhi, and J. G. Jimenez, "Edge detection algorithm based on quantum superposition principle and photons arrival probability," Int. J. Electr. Comput. Eng., vol. 10, no. 2, p. 12, 2020. DOI: 10.11591/ijece.v10i2.pp1655-1666

[31] W. K. Kong and D. Zhang, "Accurate iris segmentation based on novel reflection and eyelash detection model," in Proceedings of 2001 International Symposium on Intelligent Multimedia, Video and Speech Processing. ISIMP 2001 (IEEE Cat. No.01EX489), 2001, pp. 263-266, doi: 10.1109/ISIMP.2001.925384.

[32] J. Daugman, "New Methods in Iris Recognition," IEEE Trans. Syst. Man Cybern. Part B Cybern., vol. 37, no. 5, pp. 1167-1175, Oct. 2007, doi: 10.1109/TSMCB.2007.903540.

[33] M. N. Do and M. Vetterli, "Contourlets: a new directional multiresolution image representation," in Conference Record of the Thirty-Sixth Asilomar Conference on Signals, Systems and Computers, 2002., Nov. 2002, vol. 1, pp. 497-501 vol.1, doi: 10.1109/ACSSC.2002.1197232.

[34] P. J. Burt and E. H. Adelson, "The Laplacian Pyramid as a Compact Image Code," in Readings in Computer Vision, San Francisco (CA): Morgan Kaufmann, 1987, pp. 671-679.

[35] A. D. Rahulkar, L. M. Waghmare, and R. S. Holambe, "A new approach to the design of hybrid finer directional wavelet filter bank for iris feature extraction and classification using k-out-of-n:A postclassifier," Pattern Anal. Appl., vol. 17, no. 3, pp. 529-547, Aug. 2014, doi: 10.1007/s10044-013-0334$\mathrm{x}$.

[36] I. A. E. Rube, M. Abou, E. Nasr, M. M. Naim, and M. Farouk, Contourlet versus Wavelet Transform for a Robust Digital Image Watermarking Technique. .

[37] C. E. Shannon, "A mathematical theory of communication," Bell Syst. Tech. J., vol. 27, no. 3, pp. 379-423, Jul. 1948, doi: 10.1002/j.1538-7305.1948.tb01338.x.

[38] A. Ghaffari, M. Zarachoff, A. Sheikh-Akbari, and E. Shaghouei, "Intensity Separation based Iris Recognition Method using Curvelets and PCA," in 2019 8th Mediterranean Conference on Embedded Computing (MECO), Jun. 2019, pp. 1-5, doi: 10.1109/MECO.2019.8760170.

[39] L. Mansoura, A. Noureddine, O. Assas, and A. Yassine, "Biometric recognition by multimodal face and iris using FFT and SVD methods With Adaptive Score Normalization," in 2019 4th World Conference on Complex Systems (WCCS), Apr. 2019, pp. 1-5, doi: 10.1109/ICoCS.2019.8930748. 
[40] P. Radu, K. Sirlantzis, G. Howells, S. Hoque, and F. Deravi, "A colour iris recognition system employing multiple classifier techniques," Electron. Lett. Comput. Vis. Image Anal., vol. 12, no. 2, pp. 54-65, 2013.

[41] J. de M. Jr, H. V. Neto, E. B. Neves, and F. K. Schneider, "Biometric-oriented Iris Identification Based on Mathematical Morphology," 80(2), 181-195. DOI 10.1007/s11265-013-0861-0

[42] A. Radman, K. Jumari, and N. Zainal, "Fast and reliable iris segmentation algorithm," IET Image Process, vol. 7, p. 8, 2013. DOI: 10.1049/iet-ipr.2012.0452

[43] Mohd. T. Khan, D. Arora, and S. Shukla, "Feature extraction through iris images using 1-D Gabor filter on different iris datasets," in 2013 Sixth International Conference on Contemporary Computing (IC3), Aug. 2013, pp. 445-450, doi: 10.1109/IC3.2013.6612236.

[44] A. Abanay, Lh. Masmoudi, A. Elharif, M. Gharbi, and B. Bououlid, "Design and development of a mobile platform for an agricultural robot prototype," in Proceedings of the 2nd International Conference on Computing and Wireless Communication Systems - ICCWCS'17, Larache, Morocco, 2017, pp. 1-5, doi: 10.1145/3167486.3167527. 